\title{
Deformaciones y contagios. La lucha contra el pian en Colombia en la primera mitad del siglo XX
}

Resumen: El pian es una enfermedad contagiosa prácticamente olvidada en la actualidad. Este trabajo analiza las campañas que el gobierno colombiano puso en marcha para combatirla durante la primera mitad del siglo XX y examina las dimensiones que la enfermedad alcanzó a lo largo del país. La alta contagiosidad del pian era bien conocida, pero los factores biológicos no son suficientes para explicar su expansión. El análisis pone en evidencia las condiciones sociodemográficas de la población, los factores sociales y culturales que contribuyeron a la diseminación de la enfermedad y las vicisitudes administrativas y de diseño que enfrentaron estas campañas sanitarias.

Palabras clave: pian, enfermedad transmisible, Colombia, lucha contra las enfermedades.

\section{Deformations and contagions. The fight against yaws in Colombia in the first half of the 20th century}

Abstract: Yaws is a contagious disease currently forgotten. Yaws' high infectivity was well known; however, biological factors are not enough to explain its spread. This paper analyzes the Colombian government campaigns launched to combat yaws during the first half of the 20th century. The analysis highlights the socio-demographic conditions of the population and the social and cultural issues that contributed to the spread of the disease, and the administrative and planning vicissitudes that these health campaigns faced. It also examines the dimensions that the disease reached throughout the country.

Keywords: yaws, infectious diseases, Colombia, disease control.

\section{Deformações e contágios. A luta contra a bouba na Colômbia na primeira metade do século XX}

Resumo: A bouba é uma doença contagiosa praticamente esquecida hoje. Este trabalho analisa as campanhas que 0 governo colombiano lançou para combatê-la durante a primeira metade do século XX e examina as dimensões que a doença atingiu em todo o país. A alta contagiosidade da bouba era bem conhecida, mas os fatores biológicos não são suficientes para explicar sua disseminação. A análise destaca as condições sociodemográficas da população, os fatores sociais e culturais que contribuíram para a propagação da doença e as vicissitudes administrativas e de projeto enfrentadas por essas campanhas sanitárias.

Palavras-chave: bouba, doença transmissível, Colômbia, combate às doenças.

Cómo citar este artículo: Gabriel Jaime Vélez Tobón y Victoria Estrada Orrego, "Deformaciones y contagios. La lucha contra el pian en Colombia en la primera mitad del siglo XX", Trashumante. Revista Americana de Historia Social 18 [2021]: 28-51.

DOI: 10.17533/udea.trahs.n18a02

Fecha de recepción: 18 de junio de 2020

Fecha de aprobación: 17 de diciembre de 2020

Gabriel Jaime Vélez Tobón: Maestro en Microbiología y Bioanálisis y en Historia por la Universidad de Antioquia. Investigador en el Grupo Malaria, Universidad de Antioquia. ORCID: 0000-0002-4859-1124.

Correo electrónico: gabrielj.velez@udea.edu.co

Victoria Estrada Orrego: Doctora en Historia por la École des Hautes Études en Sciences Sociales [Francia]. Docente de la Institución Universitaria ITM [Colombia]. ORCID: 0000-0002-8872-3320.

Correo electrónico: victoriaestrada@itm.edu.co

$\cdot$ 


\title{
Deformaciones y contagios. La lucha contra el pian en Colombia en la primera mitad del siglo XX
}

\author{
Gabriel Jaime Vélez Tobón y Victoria Estrada Orrego
}

\section{Introducción}

E las últimas tres décadas, el campo de la historia de la salud y la enfermedad Lha cobrado importancia en la historiografia latinoamericana. Las perspectivas y enfoques son diversos, buscan hacer a un lado las grandes narrativas y privilegian los estudios históricos específicos. Entre ellos, hay trabajos novedosos que analizan con atención las propuestas y acciones realizadas para prevenir, combatir y erradicar enfermedades agudas, crónicas, endémicas o epidémicas. ${ }^{1}$

La historiografía colombiana también ha hecho algunos aportes a este campo y cuenta con estudios sobre la enfermedad en su singularidad espacio-temporal que examinan los discursos y las prácticas de atención, curación y prevención. Vale la pena destacar aquellos dedicados al análisis de campañas de orden nacional cuyo funcionamiento estuvo en marcha durante varias décadas. Por ejemplo, los trabajos consagrados a la viruela muestran no solo su impacto a finales del periodo colonial, el estado del saber sobre la enfermedad, los esfuerzos oficiales por contener la epidemia y la introducción de la vacuna en $1804,{ }^{2}$ sino también las técnicas de vacunación a lo largo del siglo XIX y cómo esta práctica contribuyó a construir un orden social y político moderno. ${ }^{3}$ Aquellos trabajos interesados en la lepra exponen la diversidad de estrategias para enfrentarla, que van desde el desconocimiento de la enfermedad, hasta la deshospitalización, según la noción de "curado social",

1. Carolina Biernat y Karina Ramacciotti, Historia de la salud y la enfermedad. Bajo la lupa de las ciencias sociales (Buenos Aires: Editorial Biblos, 2014); Gilberto Hochman y otros, orgs., Patologías de la patria: enfermedades, enfermos y nación en América Latina (Buenos Aires: Lugar Editorial, 2012); Diego Armus y Adrián López Denis, "Disease, Medicine, and Health", The Oxford Handbook of Latin American History, ed. José C. Moya (Nueva York / Oxford: Oxford University Press, 2010) 424-453.

2. Renán Silva, Las epidemias de viruela de 1782 y 1802 en el Virreinato de Nueva Granada (Medellín: La Carreta Editores, 2007).

3. Diana Obregón Torres, "La vacuna o la política de las cosas", Proyecto ensamblado en Colombia, t. 1, ed. Olga Restrepo Forero (Bogotá: Universidad Nacional de Colombia, 2013) 145-164. 
pasando por la segregación de los enfermos en leprosarios y la constitución de una economía de la salud. ${ }^{4}$

En lo relativo a las endemias del siglo XX, están los trabajos sobre la anemia tropical o uncinariasis. En ellos se demuestra que en Colombia la campaña antianémica (1919-1934) se presentó en el marco de la inserción del país en la economía mundial mediante las exportaciones primarias que comprometieron una inmensa parte de la población rural. La producción de café, pilar de la economía nacional, fue determinante a comienzos del siglo XX para que, sumada a los intereses de las élites locales, la Fundación Rockefeller apoyara la campaña. ${ }^{5}$ Otra endemia que ha sido objeto de interés por parte de los historiadores colombianos es la fiebre amarilla, una enfermedad que era de registro obligatorio en los puertos, y cuyos brotes epidémicos alertaron a las autoridades locales y atrajeron la atención de funcionarios de la Rockefeller. ${ }^{6}$ Aunque el funcionamiento de la campaña nacional no ha sido estudiado de forma exhaustiva (1916-1948), se ha establecido que, pese a no ser a un problema de salud pública en el país, la Fundación Rockefeller ejerció presiones para convertir la lucha contra la fiebre amarilla en una prioridad nacional, en detrimento de acciones de salud pública más urgentes para la población, ${ }^{7}$ entre ellas, la enfermedad que se analizará aquí.

Desde esta línea de estudios sobre la enfermedad, este trabajo busca hacer un aporte a la historiografia nacional y latinoamericana, al interesarse en una dolencia de la que no existe ningún estudio histórico reciente. En Colombia, el pian era una enfermedad endémica y las autoridades sanitarias nacionales dedicaron importantes esfuerzos a combatirla; se estima que durante la primera campaña nacional (1925-1931) fueron tratados más de 19,000 pacientes. Pese a la magnitud del problema de salud pública que el pian significó en toda la frontera de colonización, es el gran ausente en la historiografia de la enfermedad en Colombia. Dos factores parecen haber ayudado a silenciarlo: que no tenga relevancia en el discurso médico actual y el ser una enfermedad con bajas tasas de mortalidad. Este artículo lo saca del olvido mediante el análisis de las prácticas y los saberes médicos que contribuyeron a desarrollar la campaña antipiánica colombiana en la primera mitad del siglo XX. La investigación de archivo permitió conocer las discusiones planteadas desde la medicina para enfrentar una problemática nacional, y pone en evidencia

4. Diana Obregón Torres, Batallas contra la lepra: Estado, medicina y ciencia en Colombia (Medellín: Fondo Editorial Universidad EAFIT / Banco de la República, 2002); Abel Martínez y Samuel Guatibonza, "Cómo Colombia logró ser la primera potencia leprosa del mundo: 1869-1916", Colombia Médica 36.4 (2005): 244-253.

5. Emilio Quevedo y otros, Café y gusanos, mosquitos y petróleo: el tránsito desde la higiene hacia la medicina tropical y la salud pública en Colombia, 1873-1953 (Bogotá: Universidad Nacional de Colombia, 2004); Claudia Mónica García y Emilio Quevedo, "Uncinariasis y café: los antecedentes de la intervención de la Fundación Rockefeller en Colombia: 1900-1920”, Biomédica 18.1 (1998): 5-21.

6. Quevedo y otros 271-288.

7. Paola Mejía Rodríguez, "De ratones, vacunas y hombres: el programa de fiebre amarilla de la Fundación Rockefeller en Colombia, 1932-1948”, Dynamis 24 (2004): 119-155. 
las condiciones materiales y sociodemográficas de las poblaciones víctimas de esta endemia, así como las estrategias y acciones de las autoridades sanitarias movilizadas a las selvas para controlar la enfermedad.

\section{Conocimiento médico sobre el pian}

Es necesario hacer algunas precisiones sobre esta dolencia poco conocida actualmente. El pian es una enfermedad infecciosa crónica ocasionada por la bacteria Treponema pallidum subespecie pertenue, y se caracteriza por la erupción de lesiones en piel que pueden estar acompañadas de dolores reumáticos y, en los casos más graves, de lesiones óseas deformantes. ${ }^{8}$ En 1920, algunos funcionarios de higiene colombianos se referían a ella como "una enfermedad que repugna cuando se ve por primera vez y que siempre impresiona desagradablemente. Es muy contagiosa y con mucha frecuencia se propaga cuando hay comercio entre poblaciones, en donde no ha existido"?

Según las características clínicas y la sintomatología del enfermo, el pian fue delimitado por los médicos en tres periodos. El periodo primario estaba caracterizado por la aparición de una pequeña lesión cutánea llamada "buba madre", acompañada por dolores reumáticos y fiebres (Figura 1). Entre dos y cuatro semanas después, se presentaba la segunda etapa, o una forma diseminada, en la cual brotaban en la piel múltiples lesiones (Figura 2); durante esta fase, los pacientes podían sufrir una de las formas más incapacitantes de la enfermedad, en la que aparecían lesiones plantares de manos y pies, estos últimos eran denominados "clavos" o "empedraduras" y, además de dolorosos, eran difíciles de eliminar con los tratamientos habituales. Cuando el clavo se presentaba en ambas piernas, el enfermo se veía obligado a mantenerse sentado y a desplazarse sobre las nalgas, con ayuda de los brazos. Finalmente, cuando la enfermedad tomaba un carácter crónico y no se asistía a ningún tratamiento, aparecía la forma más grave o pian terciario. Este periodo se caracterizaba por la alteración del aspecto físico — producía incapacidad al afectar la piel, los cartílagos, los tendones y los huesos- y un amplio espectro de formas clínicas en el cual eran comunes las deformidades óseas, las ulceraciones profundas y la destrucción del tabique nasal, entre otras (Figura 3). ${ }^{10}$

8. Había una amplia sinonimia para referirse a esta enfermedad. Algunos de los nombres eran: bubas en Venezuela, Antillas y Brasil; bobas en el archipiélago de las Azores; buba en el África Central. Los alemanes y holandeses la llaman frambuesia tropical. Los franceses, pian, y los ingleses, yaws. En Colombia se nombraba en la mayoría de las regiones como pian. Esta denominación era utilizada sobre todo en la región sur de la costa del Pacífico, es decir, en Nariño, Cauca y Valle. Otro apelativo común en el país era el de buba o bubas, usado con frecuencia hacia el norte de la costa pacífica, es decir, al interior del Chocó y el golfo de Urabá.

9. Jesús M. Marulanda, "Informe que presenta el secretario de gobierno al señor general Pedro Nel Ospina Gobernador del departamento al reunirse la asamblea de 1920", Medellín, 1920. Archivo Histórico de Antioquia (AHA), Medellín, Impresos y publicaciones, 110.

10. Gerardo López Narváez, "Pian y campaña antipiánica en Colombia”, Revista de la Facultad de Medicina (Bogotá) abril de 1956: 294-342. 

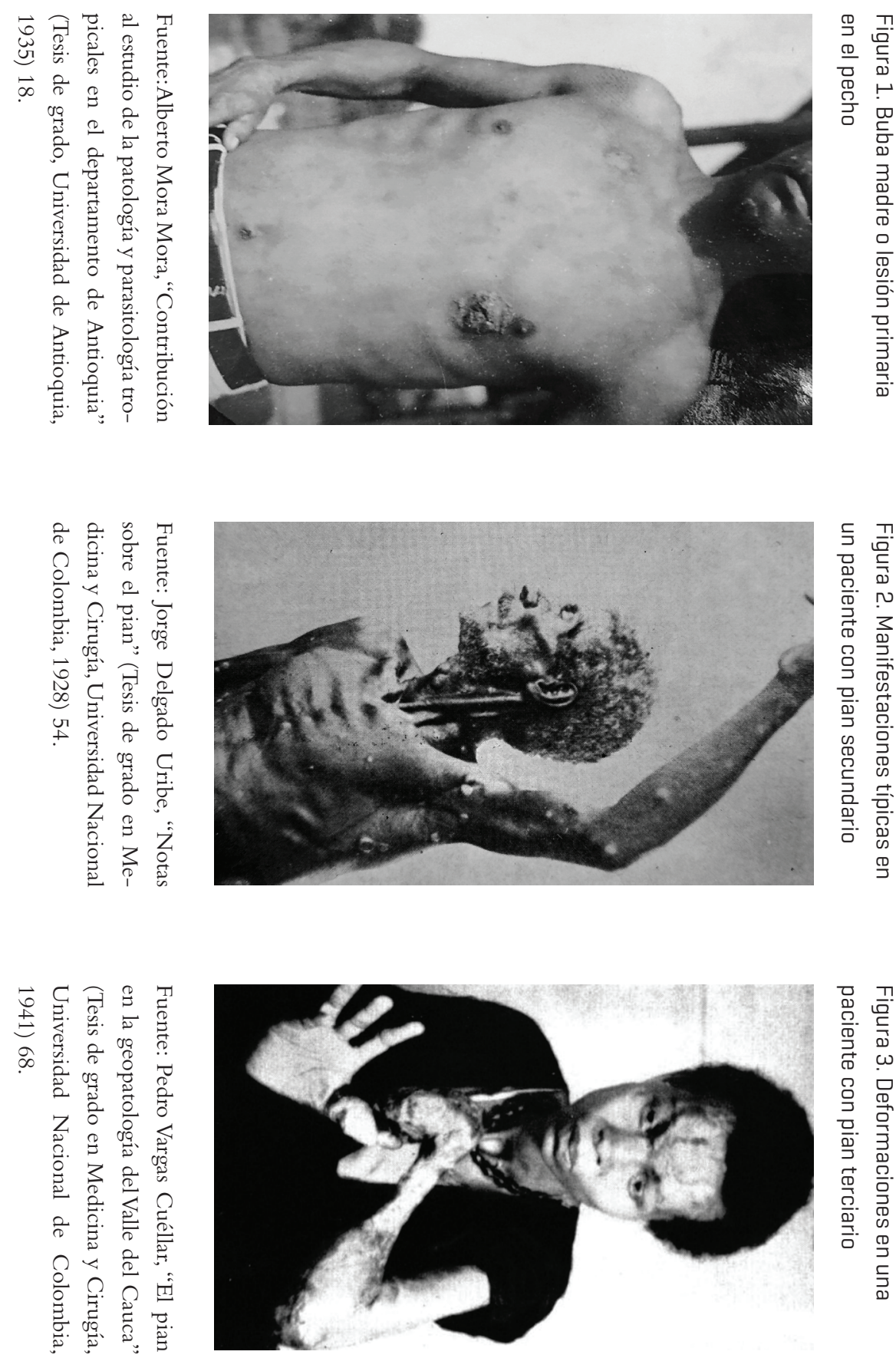


\section{Tras las huellas del pian en Colombia}

Los primeros estudios sobre el pian en Colombia fueron el resultado de un intercambio epistolar, a finales del siglo XIX y comienzos del XX, entre médicos locales que trabajaban fuera de la capital, en departamentos como Antioquia y Boyacá. En sus cartas, relataban la aparición de epidemias de bubas y de otras enfermedades imperantes en las localidades donde ejercían la profesión. ${ }^{11}$

Destaca el trabajo del médico Miguel Canales, ${ }^{12}$ quien en 1894 realizó una descripción clínica de la enfermedad y estableció diferencias entre las lesiones ocasionadas por el pian y la sífilis, que eran motivo de confusión para los médicos de la época. ${ }^{13}$ Canales también señaló el inconveniente de que personas enfermas y sanas convivieran bajo el mismo techo, dada la contagiosidad de esta patología. En 1889, el trabajo sobre "Puercas o marranas", ${ }^{14}$ de Indalecio Camacho, complementaba las observaciones clínicas de Canales. ${ }^{15}$ Uno de los trabajos mejor documentados sobre el pian fue publicado en 1910 por Gabriel Toro Villa, a partir de sus observaciones en las minas del Muzo, del departamento de Boyacá, en el centro del país, donde se trasladó, junto con otros médicos reconocidos, para atender una epidemia de fiebre amarilla que se había presentado alli. ${ }^{16}$

Durante la época, la salud era parte de la cartera del Ministerio de Educación, que buscaba educar al pueblo sobre las enfermedades dominantes en el territorio nacional y dar instrucciones para enseñar a combatirlas. Por ejemplo, la revista Instrucción Pública Antioqueña tenía una sección para la vulgarización de los principios generales de higiene, donde se contemplaba la profilaxis de enfermedades que afectaban al departamento. ${ }^{17}$

Para el año 1911, la revista ya advertía sobre la propagación "alarmante" del pian en el occidente de Antioquia. Incluso se clausuraron escuelas y se enfatizó en la susceptibilidad de los niños a la infección, debido a la fragilidad de su piel, que no siempre estaba bien cubierta, y a su propensión a los traumatismos, que permitían la entrada de la bacteria. Se insistió en limitar la infección a través de medidas

11. Eduardo Kairuz, "Contribución al estudio del pian" (Tesis de doctorado en Medicina y Cirugía, Universidad Nacional, 1938) 37.

12. Citado por Kairuz 37.

13. Producto de la confusión acerca de si el pian y la sífilis eran o no la misma enfermedad, surgieron dos corrientes médicas: unicistas y dualistas. Para los primeros, el pian y la sífilis eran una misma enfermedad y uno de sus principales argumentos era que el tratamiento yodo-mercurial era igualmente efectivo. Para los dualistas, la sífilis y el pian eran entidades completamente diferentes tanto por el agente infeccioso como por sus signos y síntomas. Kairuz 32.

14. El término se refiere a la manera en que era nombrada la leishmaniosis en ese tiempo en Boyacá.

15. Kairuz 37.

16. Gabriel Toro Villa, "Bubas, frambuesia tropical”, Repertorio de Medicina y Cirugía (Bogotá) junio de 1910: 515-538.

17. Nepomuceno Jiménez, "Algo sobre higiene. Bubas", Instrucción Pública Antioqueña (Medellín) agosto de 1911: 884 . 
higiénicas como tratar las heridas cutáneas con productos antisépticos, cubrir la herida con emplastos y bañarse a diario en aguas diferentes a aquellas que servían a la población sana. ${ }^{18}$

Debido a la alta contagiosidad de la enfermedad, en Antioquia ya se había planteado la conveniencia del aislamiento de los pianosos. Por un lado, había detractores de esta idea, como el doctor Nepomuceno Jiménez, quien veía esta medida no solo como innecesaria, dada la baja mortalidad de la enfermedad, sino también como impracticable, debido a los gastos que suponía confinar a los enfermos. ${ }^{19}$ Otros, como el director departamental de Higiene de Antioquia, Juan B. Londoño, defendían el aislamiento y proponían la creación de hospicios para los pianosos pobres y los niños enfermos, con personal para atenderlos y brindarles alimentación y tratamiento. ${ }^{20}$

Estas publicaciones de instrucción pública también intentaron combatir nociones erróneas sobre la enfermedad, ya que muchos enfermos tenían prácticas que atentaban contra la higiene y favorecían su propagación. Por ejemplo, entre los pianosos se pensaba que mojar las bubas era inconveniente y que era mejor tratarlas después de cierto tiempo. Como muchos pacientes no lograban acceder al tratamiento, ${ }^{21}$ y sus lesiones tendían a una larga cronicidad, entonces existía entre ellos la creencia de que las bubas duraban siete años, con brotes anuales. ${ }^{22}$

La instrucción higiénica era de importancia en las escuelas, pues se consideraba que el pian podía desembocar en hábitos faltos a la moral. Los niños debían evitar sucumbir a contraer hábitos de holgazanería difíciles de desarraigar que derivarían en individuos problemáticos para la sociedad propensos a la cárcel. Igualmente, se argumentaba que los adultos enfermos tendían a la pereza debido a la larga incapacidad, infectaban a sus familias y las abandonaban a la miseria. Se convertían en mendigos, recorrían pueblos vecinos y expandían la enfermedad, despertaban compasión y hacían gala de su "repugnante condición". 23

Durante la primera mitad del siglo XX, el discurso higiénico estaba estrechamente ligado con las ideas de moralidad y de progreso. La comunidad médica promulgaba que los grandes problemas que afectaban a la higiene eran, además de las enfermedades venéreas como la sífilis y la blenorragia, comportamientos que atentaban contra la moral, como el alcoholismo, las uniones maritales ilegítimas, la holgazanería, entre otros.

Para la década de 1920, la distribución del pian en el territorio nacional era conocida. Según el médico Antonio José Rodríguez, se encontraba en todas las

18. Jiménez 887.

19. Jiménez 887.

20. Juan B. Londoño, "Sobre tratamiento del pian, o sea las bubas", Instrucción Pública Antioqueña (Medellín) enero-marzo de 1917: 254.

21. Los aspectos relacionados con el tratamiento serán explicados más adelante.

22. Jiménez 886-887.

23. Jiménez 887-888. 
regiones cálidas y húmedas del país y si se descuidaban los focos esporádicos al interior de la nación se propagaría con facilidad. ${ }^{24}$ La enfermedad se extendía a largo de las hoyas hidrográficas de ríos como el Magdalena, el Cauca, el Sinú y el Atrato. Esto abarcaba regiones del litoral Pacífico, Urabá, municipios de la costa norte y zonas limítrofes con Venezuela. Sin embargo, también se podían presentar focos de la enfermedad fuera del litoral Pacífico y del Magdalena o sus principales afluentes. ${ }^{25}$

\section{La vida de los pianosos}

En la década de 1920, la alta contagiosidad de la enfermedad llamó la atención de la Dirección Nacional de Higiene, que recibía constantemente telegramas en los que se reclamaba el envío de médicos hacia las regiones para combatir el pian. Asimismo, se presentaban situaciones que favorecían el contagio, como, por ejemplo, cuando un enfermo dormía de manera transitoria en alguna posada y otro viajero la contraía al pernoctar en el mismo sitio. ${ }^{26}$ El médico Samuel Pérez Mejía relata que la llegada de las bubas al departamento de Caldas fue producto de una mujer, quien adquirió la infección en Puerto Berrío, Antioquia y, al llegar a la provincia de Manzanares en Caldas, llevó la epidemia y la propagóo. ${ }^{27}$

Para los médicos era preocupante la forma en que se mezclaban las personas sanas y las enfermas con pian en las actividades cotidianas, pese a que era considerada como una enfermedad repugnante; esto denotaba la naturalidad y ausencia de miedo hacia el pian. Era común entre los pobladores compartir la morada, incluso la cama, con algún pianoso, o alimentarse del mismo plato. De igual forma, y en contravención de las recomendaciones médicas, muchos niños pianosos asistían a las escuelas. ${ }^{28}$

Las autoridades sanitarias nacionales veían a la enfermedad como una condición asociada a la pobreza y la miseria. Una adecuada higiene era fundamental para prevenir la infección y, de hecho, era poco habitual ver a una persona de cierta posición social enferma de pian. Los trabajos de medicina consagrados al pian, escritos en la primera mitad del siglo XX, describían la enfermedad e incluían causas y la patogenia misma; buscaban entenderla y asociarla a factores adyuvantes

24. Antonio José Rodríguez R., "El pian en Colombia”, Revista de Medicina y Cirugía (Barranquilla) 1939: 23.

25. Ernesto Arango, Contribución al estudio de enfermedades tropicales: pian (Tesis de doctorado, Universidad de Antioquia, 1922) 38-39.

26. Jorge Delgado Uribe, “Notas sobre el pian” (Tesis de grado en Medicina y Cirugía, Universidad Nacional de Colombia, 1928) 59-60.

27. A pesar de no encontrar la referencia directamente del médico Samuel Pérez Mejía, este hecho es relatado en: Rodríguez R. 23; Felix Henao Toro, "El pian en el Departamento de Caldas", Revista de Higiene de Bogotá (Bogotá) 1936: 121.

28. Alberto Mora Mora, "Contribución al estudio de la patología y parasitología tropicales en el departamento de Antioquia" (Tesis de grado, Universidad de Antioquia, 1935) 16. 
y predisponentes. Como causas adyuvantes, había un consenso en cuanto a los modos de vida y las costumbres de las personas que las hacían más susceptibles a desarrollar la enfermedad. Dicha susceptibilidad estaba relacionada con condiciones internas y externas que afectaban al cuerpo del individuo, ${ }^{29} \mathrm{y}$ uno de los principales factores era el clima tropical, tanto, que los médicos resaltaron que en los climas fríos la enfermedad no se presentaba, mientras que la infección se acentuaba con el aumento del calor. ${ }^{30}$

La distribución de la enfermedad también se asoció a los modos de vida de los habitantes de las regiones en las que había focos de pian. La desnudez en las actividades cotidianas y la falta de higiene de los individuos fueron vistos como factores que potenciaron las infecciones piánicas. Además, la mala alimentación y el paludismo constante predisponían los cuerpos a una extrema debilidad que los hacía más susceptibles a la bacteria. ${ }^{31}$

Durante las campañas contra el pian en el Cauca en la década de 1940, el médico Pedro Vargas Cuéllar señalaba dos aspectos esenciales que hacían más persistente la enfermedad: la profesión y la alimentación. Según él, "la gravedad de la enfermedad crece con el ascenso por los ríos y las dificultades de alimentación", ${ }^{32}$ pues los negros que habitaban las orillas de los ríos y vivían de la minería o de una agricultura incipiente tenían un régimen alimenticio pobre que los hacía propensos a desarrollar grandes lesiones, ulceraciones y diseminación de las bubas por todo su cuerpo. Por el contrario, aquellos dedicados a la pesca a orillas del mar comían pescado en abundancia y quienes trabajaban en el Ferrocarril del Pacífico tenían una buena alimentación, pues ganaban un mejor sueldo, por lo que no tendían a sufrir lesiones óseas y presentaban menos complicaciones o secuelas después del tratamiento de las bubas. Además de estos aspectos, también había prácticas de los pobladores de zonas endémicas que aumentaban de forma considerable la persistencia de la enfermedad y dificultaban el trabajo médico. La concepción fatalista de algunas comunidades hacía que la adquisición y perpetuación del pian fuera inevitable. Al respecto, un médico relataba:

Entre nosotros (auncuando [sic] realmente, por fortuna tan irracional práctica ya se está abandonando) la transmisión en muchos casos se hacía voluntariamente: con la uña o con cualquier objeto vulnerante se hacía sobre la piel sana de los niños casi recién nacidos una escoriación sobre la cual se frotaba las costra [sic] de una lesión evolutiva. Esto con el objeto de buscar alguna suerte de inmunidad y sobre todo según mi personal observación porque entre las gentes de raza

29. Había un consenso sobre la falta de aseo como factor predisponente para adquirir la enfermedad. Marulanda 111; Arango 17.

30. Arango 17.

31. Eduardo Vasco Gutiérrez, "Espiroquetosis cutánea (pian) y su tratamiento por el muthanol y por el storvarsol" (Tesis de grado, Universidad de Antioquia, 1925) 13-15; Arango 13-17; Mora Mora 15-17.

32. Pedro Vargas Cuéllar, "El pian en la geopatología de la Costa delValle del Cauca" (Tesis de grado en Medicina y Cirugía, Universidad Nacional de Colombia, 1941) 28. 
negra pobladoras de las regiones en que el Pian es endémico había y aún hay respecto de él un concepto fatalista: pensaban que por pertenecer a tal raza era inevitable que en alguna época de la vida la padecerían. ${ }^{33}$

También se creía que un pianoso podía lograr que sus lesiones emigraran a una persona sana y curarse. El médico Eduardo Vasco Gutiérrez explicaba la práctica:

\footnotetext{
Si se recogen en un papel tantas piedrezuelas cuantas verrugas tiene un individuo, piedras con las cuales debe tocarse previamente la verruga, y luego se arroja a la calle el saquito de papel, el que lo recoja y toque las piedras se llenará de papilomas mientras que el otro quedará curado de ellas. También, pues, emigran las verrugas. ${ }^{34}$
}

Con base en esta creencia, el médico citaba a un sacerdote de nombre Greggio, quien mencionaba que en el Congo Belga una tribu libre de enfermedad había sido contaminada por una tribu vecina a través del pan. Pequeñas costras provenientes de las lesiones pianosas se ocultaron en trozos de pan, para luego ser vendidos a la tribu vecina, pues se pensaba que, si la enfermedad era adquirida por los compradores, esta desaparecería de los vendedores. ${ }^{35}$

\section{Tratamiento y disputas médicas}

Al tener en cuenta la capacidad de propagación de la enfermedad, una vez que era identificado un foco de pian, era clave dar tratamiento para contener la expansión y proteger a la población sana. Antes de la aparición de los antibióticos, los medicamentos utilizados para curar el pian podrían dividirse en tres grupos principales: los arsenicales, los bismúticos y los yodurados. ${ }^{36}$

Los medicamentos más usados tenían como base farmacológica el arsénico. A este grupo pertenecían el salvarsán y el neosalvarsán. Si bien el salvarsán eliminaba los treponemas, su uso era complejo por su baja solubilidad, de allí que no fuera bien tolerado por el organismo. Además, se requería de un proceso químico de alcalinización que complejizaba su manipulación, detalle clave, pues con frecuencia las dosis del tratamiento eran administradas por boticarios o inspectores sanitarios poco calificados. ${ }^{37}$

Como alternativa estaba el neosalvarsán o 914, que tenía mayor solubilidad y no necesitaba procedimientos previos para inyectarlo. Dadas sus propiedades solubles, era posible inyectarlo directamente en sangre sin peligro de causar daño a quienes

\section{López Narváez 294-342.}

34. Vasco Gutiérrez 14-15.

35. Vasco Gutiérrez 15.

36. Florencio Nieto Escobar, "Algunas observaciones sobre el pian en la región de Medina" (Tesis de grado en Medicina y Cirugía, Universidad Nacional de Colombia, 1945) 35.

37. Nieto Escobar 35-36. 
lo recibían. ${ }^{38}$ El 914 fue el medicamento más usado en las campañas antipiánicas en Colombia, pues se consideraba el más efectivo y específico para la curación de las lesiones. ${ }^{39}$ El bismuto fue otro de los medicamentos de uso extendido; pese a no ser tan efectivo como los arsenicales, permitía una buena eliminación de las lesiones en piel. ${ }^{40}$ Finalmente, los yoduros se usaban como medicamento auxiliar. ${ }^{41}$

Aunque el neosalvarsán era el de mayor efectividad, su aplicación no estuvo exenta de accidentes, en ocasiones con desenlace fatal. Según el médico Eduardo Vasco Gutiérrez, los enfermos veían el 914 como una especie de panacea universal y desestimaban los cuidados que debían tenerse para su aplicación. En las regiones invadidas por el pian, el medicamento en ocasiones era aplicado por boticarios o curanderos, quienes creían que la droga era inocua y que, entre más alta fuera la dosis, mayor beneficio traía:

Gentes del pueblo, sugestionadas con los milagros del 914, se fueron al boticario, le compraron la mayor ampolla que hubiera y se la hicieron aplicar allí mismo.Y el boticario, que para el caso todos son uno, quien cree que todas las ampollas se pueden aplicar indistintamente e ignora toda base científica, las aplica sin ningún examen previo. Ambos pagaron con la vida su fervor por la droga: el uno en el momento en que se la aplicaban, el otro algunos días después. ${ }^{42}$

El neosalvarsán no siempre era efectivo en los casos más crónicos, razón por la cual en muchas ocasiones se recurría a hacer combinaciones con las otras drogas disponibles. ${ }^{43}$ Como se verá más adelante, la llegada de la penicilina al país representó el cambio definitivo en el tratamiento. En la campaña nacional de 1950, una de las razones de la transición de los arsenicales a la penicilina fue la dificultad de tratamiento en los casos reacios o resistentes.

Aunque en la mayoría de los casos el tratamiento era efectivo, la falta de medicamentos y de médicos oficiales fue una problemática constante para la eliminación de focos pianosos en el país. ${ }^{44}$ En 1919, el alcalde de Sopetrán, municipio del occidente antioqueño, informó a las autoridades sanitarias que el tratamiento había dado un magnífico resultado, pero señaló que faltaban 200 bubosos por ser atendidos. Un segundo envío con 90 dosis también resultó insuficiente. ${ }^{45}$

Se presentaron también casos en los cuales se enviaban dosis, pero no había un médico oficial, así que el tratamiento debía ser aplicado por el médico de

38. El uso del 914 era tóxico en individuos con insuficiencias hepáticas y otras enfermedades, esto suponía conocimientos de base para utilizarlo. Véase Nieto Escobar 36-39.

39. Marulanda 109-111. Esta información es también mencionada en: Delgado Uribe 47.

40. Nieto Escobar 39-46.

41. Vargas Cuéllar 43-44.

42. Vasco Gutiérrez 35.

43. Delgado Uribe 47-48.

44. Los médicos oficiales eran funcionarios pagados con erarios de cada municipio.

45. Marulanda 111. 
otro municipio, lo que retrasaba el procedimiento. La falta de médicos oficiales en zonas rurales de Colombia incrementó la deficiente atención en salud; para el año 1923, se denunciaba la ausencia de médicos oficiales que contribuyeran a la producción de estadísticas en salud. Existían problemas estructurales desde la norma que regulaba las condiciones para sostener los médicos oficiales, lo que, sumado al reducido número de médicos graduados, afectaba la atención en salud en el territorio rural. ${ }^{46}$ En regiones en las que había un médico oficial, algunos se encontraron con panoramas que impedían sus labores. En 1922, el médico del Bajo Cauca, Salvador Jaramillo, denunció que en algunos municipios, además de "la desidia y desaseo de los campesinos", sus habitantes eran supersticiosos y tenían miedo a los tratamientos médicos:

A pesar de los esfuerzos hechos para extirpar los focos endémicos de pian existentes en algunos parajes, son muchos los casos que permanecen sin tratamiento porque en ninguno de ellos hay autoridades que apliquen la respectiva sanción, tan necesaria en estos casos, máxime si se tiene en cuenta que los enfermos tienen la absoluta prohibición de los yerbateros de hacerse aplicar la inyección de Neosalvarsán, alegando como causa que esta no puede curarlos como las yerbas, y que además el médico trata de envenenarlos. ${ }^{47}$

Por su parte, el médico oficial Marco Robledo manifestaba una situación similar en su municipio, pues las personas buscaban más a los yerbateros que al médico oficial. Según Robledo, los yerbateros mantenían en la ignorancia a los enfermos y los manipulaban para que estos evitaran los tratamientos y las recomendaciones de la medicina universitaria. Paradójicamente, algunos yerbateros que sufrieron la enfermedad siguieron los tratamientos propuestos por los médicos diplomados (Figura 2). ${ }^{48}$ Esto es un ejemplo de la lucha por parte de los médicos graduados de la época contra los irregulares y de su búsqueda por consolidar el monopolio del ejercicio de la profesión médica. ${ }^{49}$

La presencia de los médicos en los focos endémicos de pian era transitoria y, en la mayoría de los casos, esto impedía completar los tratamientos, que consistían

46. José M. González Gómez, "Memoria que presenta el secretario de gobierno al señor doctor Ricardo Jiménez Jaramillo Gobernador del departamento al reunirse la asamblea de 1923", Medellín, 1923. AHA, Medellín, Impresos y publicaciones, 186.

47. José M. González Gómez, "Memoria que presenta el secretario de gobierno al señor doctor Ricardo Jiménez Jaramillo Gobernador del departamento al reunirse la asamblea de 1924", Medellín, 1924. AHA, Impresos y publicaciones, 203.

48. La persona que aparece en la imagen provenía del Alto Sinú y era minero y agricultor. Curiosamente, este paciente era el curandero de su región y contaba con una numerosa clientela por su éxito en el tratamiento del pian. El paciente además llegó con tres de sus hijos infectados de pian para ser tratados en el dispensario de Montería. Delgado Uribe 54-55.

49. A propósito de las disputas por la regulación del ejercicio de la medicina, véase Jorge Márquez y Victoria Estrada, "Culebrero, tegua, farmaceuta y dentista. El Indio Rondín y la profesionalización médica en Colombia, 1912-1934", Anuario Colombiano de Historia Social y de la Cultura 45.1 (2018): 79-104. 
en varias dosis. Así que los campesinos acudían a tratamientos empíricos. No obstante, Robledo señalaba optimista que muchos de los escépticos iban superando el temor infundido al ver la mejoría de sus vecinos tratados con el $914 .{ }^{50}$

\section{La primera campaña antipiánica en Colombia}

A la par que el pian se extendía en el territorio nacional, las autoridades de higiene enfrentaron diversos problemas de salud pública. En cooperación con la Fundación Rockefeller, a partir de 1920, comenzó a funcionar la campaña contra la uncinariasis en diferentes regiones del país y, en 1923, la campaña contra la fiebre amarilla, sobre todo en los puertos del Pacífico colombiano. La estrategia contra la uncinariasis consistía en el diagnóstico y el tratamiento masivo con antiparasitarios para eliminar la infección. La campaña también buscaba cambios en los hábitos sanitarios (como la implementación de letrinas en las viviendas), así como de educación, mediante charlas y repartición de folletos.

Frente a enfermedades como la tuberculosis, que había comenzado a expandirse en las costas y regiones de clima templado, la estrategia consistió en dictar disposiciones en materia profiláctica a través de conferencias públicas, educación en las escuelas y la publicación de cartillas y folletos. Asimismo, se buscó establecer dispensarios y crear auxilios para la construcción de pabellones especiales en los hospitales municipales para los tuberculosos. ${ }^{51}$

La campaña antipiánica en Colombia no solo centró sus esfuerzos en la distribución del medicamento, sino también en la creación de comisiones sanitarias en los focos endémicos del país. Antes de la expedición de la resolución 306 de 1925, de la Dirección Nacional de Higiene, la lucha contra el pian estaba limitada a algunas acciones aisladas por parte de los distritos o incluso de algunos particulares. Excepto en Antioquia, donde los primeros esfuerzos por organizar una campaña se hicieron del asocio entre organismos gubernamentales y privados. Así, el Departamento Médico del Ferrocarril de Antioquia puso a disposición de los pianosos sus hospitales y dispensarios. Por su parte, a partir de 1917, la Dirección Departamental de Higiene se encargó de la creación de dispensarios para el tratamiento de enfermedades venéreas y pian, así como del fortalecimiento de la estadística médica. Estos datos eran fundamentales para el envío de medicamentos a los focos endémicos y sirvieron de apoyo para la creación de plazas médicas específicas en el Bajo Cauca y Urabá.

En Antioquia, la campaña antipiánica tuvo un modelo centralizado, con envío de medicamentos hacia las diferentes regiones para que los médicos oficiales aplicaran los tratamientos. El registro estadístico permitió conocer el estado de la

50. José M. González Gómez, "Memoria que presenta el secretario de gobierno al señor doctor Ricardo Jiménez Jaramillo Gobernador del departamento al reunirse la asamblea de 1925”, Medellín, 1925. AHA, Medellín, Impresos y publicaciones, 210-215.

51. Pablo García Medina, "La organización sanitaria en Colombia”, Boletín de la Oficina Sanitaria Panamericana (OSP) (Washington) enero de 1927: 3-14. 
enfermedad en los municipios antioqueños; los médicos asentaban el nombre del enfermo, la edad, el sexo y el número de inyecciones empleadas hasta la total curación del individuo. ${ }^{52}$ Para 1920, se registraron 545 casos en nueve municipios. Cuatro años más tarde, la cifra ascendía a 7,162 casos, distribuidos en 29 municipios. ${ }^{53}$

Las razones para que las autoridades sanitarias decidieran emprender una campaña nacional fueron múltiples, y la alta contagiosidad fue un factor determinante. El médico Jorge Delgado Uribe señalaba que era perentorio iniciar una campaña:

Sería acaso, con un criterio indolente, hasta admisible la tesis de que cada persona pianosa disfruta del derecho de disponer de la vida y sus medios de actividad y de dedicar su cuerpo al cultivo del treponema pertenue, si ella viviera aisladamente y no amenazara a todos sus semejantes. Pero, aparte de que ello pugna con elementales principios de moral y de religión y con un concepto civilizado del estado opuesto al de un bárbaro individualismo, sobre las autoridades pesa el deber imperioso de extirpar en esas personas lo que constituye un permanente y temible peligro para la sociedad toda. ${ }^{54}$

Otra de las razones se sustentó en el hecho de que en varios países ya habían comenzado campañas con un éxito considerable. En Filipinas, durante el año 1921, se crearon dispensarios especiales para el tratamiento de bubosos y, dos años después, se observó una disminución en el número de infectados. También se tenía conocimiento de algunas campañas emprendidas en África. ${ }^{55}$

Así, para el año 1925, el director nacional de Higiene, Pablo García Medina, y Juan N. Corpas, ministro de Instrucción y Salubridad, fundaron la Comisión Nacional para Combatir el Pian, a través de la Resolución 306 de 1925. Los recursos para la financiación de la campaña corrieron por cuenta del Estado colombiano, y nombraron jefe a Salvador Jaramillo Berrío, quien ya había tenido una larga experiencia en el tratamiento de enfermos con pian como médico de la región del Bajo Cauca (Figura 4). ${ }^{56}$

El comienzo de la campaña estuvo a cargo de Jaramillo Berrío y Jorge Delgado Uribe en el río Magdalena, quienes comenzaron labores en municipios de Magdalena

52. Francisco Pérez, "Informe del secretario de gobierno presentado al señor gobernador del departamento Dr. Manuel M. Toro con motivo de la reunión de la asamblea de 1922”, Medellín, 1922. AHA, Medellín, Impresos y publicaciones, 131.

53. González Gómez 181-182.

54. Delgado Uribe 59.

55. Delgado Uribe 59-60. Muchos de estos antecedentes se basaron en información encontrada en la edición número 30.4 (1926) de The Philippine Journal of Science (Manila). En esta entrega de la revista aparecieron varios artículos sobre el pian.

56. La campaña antipiánica de 1925 se centró en atacar los focos existentes a lo largo del Río Magdalena y Cesar, además de algunas regiones en Caldas, Bolívar, Córdoba y Antioquia. Para 1936 la campaña antipiánica se desplegó en los focos de Nariño, Cauca, Valle del Cauca y Chocó. Finalmente, para 1950 además de estos departamentos del Litoral Pacífico se adhirieron Caldas y Antioquia. Kairuz 39-40; Delgado Uribe 60. 
Figura 4. Campañas antipiánicas en Colombia en la primera mitad del siglo XX

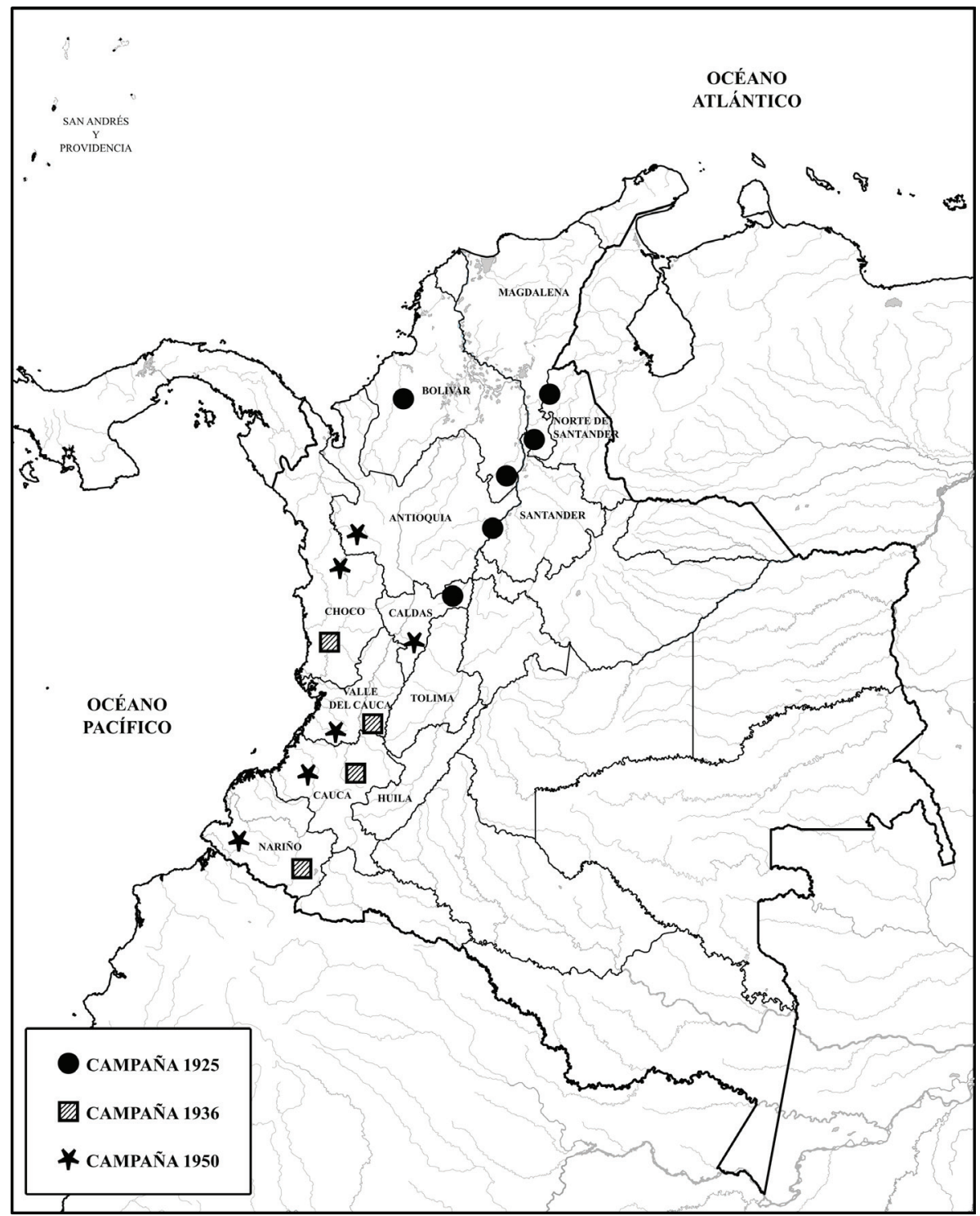

Fuente: Elaboración propia.

y Antioquia. Al año siguiente, se hizo una adición de personal y se iniciaron trabajos en los departamentos de Bolívar y Caldas. En este último se creó una comisión que también enfrentó enfermedades como el paludismo, la disentería amebiana, la uncinariasis y la leishmaniasis. Dado que muchas de las zonas con este tipo de enfermedades eran de difícil acceso, la Dirección Departamental de Higiene de Caldas dotó a la campaña de una lancha que contaba con un pequeño laboratorio bacteriológico y 
un botiquín. Esta lancha hizo nueve viajes en 1930 y dos más en los primeros meses de 1931. Algunos de esos recorridos abarcaron 1,800 kilómetros e incluyeron actividades en 14 caseríos y la atención de más de 500 enfermos ${ }^{57}$ Durante el tiempo que ejerció la Comisión Nacional para combatir el pian se hicieron algunos intentos por emprender la campaña en Nariño, pero no se consolidaron. ${ }^{58}$

En un balance de la campaña, que funcionó entre 1925 y 1931, el director nacional de Higiene, Pablo García Medina, destacó que se habían abarcado 44 regiones, correspondientes a seis municipios del país, y que habían sido atendidos 19,185 enfermos, de los cuales 12,332 se habían curado por completo; además, 3,994 trabajadores habían podido volver a retomar sus actividades. Pese a los buenos resultados, en 1931, la campaña nacional fue suspendida por falta de recursos. ${ }^{59}$

La intendencia de Chocó comenzó a llamar la atención de las autoridades sanitarias debido a la cantidad de enfermedades que afectaban a sus pobladores. En una entrevista concedida al periódico El Tiempo en 1936, el médico Antonio José Rodríguez se refería a la situación así:

Antes de 1930, ninguno de los gobernantes del Chocó, ninguno de los encargados de salubridad pública había denunciado al país la espantosa tragedia de esa Intendencia. Se ignoraba absolutamente que el pian, el paludismo, la anemia, las actinomicosis, fueran enfermedades tratables científicamente, y por tanto, hasta la misma época nada se había hecho en beneficio de las clases campesinas, de los mineros chocoanos. ${ }^{60}$

Dentro de las principales preocupaciones anotadas en los informes médicos estaba la cantidad de enfermos con pian terciario, la forma más grave e incapacitante de la enfermedad. Esto ocasionaba en muchos casos que los individuos quedaran imposibilitados para la marcha y el trabajo. Con la aplicación del tratamiento la infección desaparecía, pero las cicatrices deformantes y muchas veces incapacitantes permanecían. $^{61}$

La campaña antipiánica en el Chocó, iniciada en 1930, se caracterizó por la creación de dispensarios en diferentes regiones de la intendencia. En principio se crearon dos centros de tratamiento en las poblaciones de Quibdó e Istmina ${ }^{62}$ y posteriormente se establecieron 23 centros curativos en lugares donde confluía la población que vivía a lo largo de los ríos. De esos centros, cinco eran atendidos por médicos y los 18 restantes por inspectores sanitarios preparados por la Dirección

57. Pablo García Medina, "La sanidad en Colombia”, Boletín de la Oficina Sanitaria Panamericana (OSP) (Washington) abril de 1932: 356-357.

58. Rodríguez R. 18-20.

59. Medina 356-357.

60. “La campaña antipiánica se unificará pronto en varios departamentos”. El Tiempo (Bogotá) 8 de marzo de 1936: 7.

61. Oficina Sanitaria Panamericana, "Frambesía”, Boletín de la Oficina Sanitaria Panamericana (OSP) (Washington) 1935: 871-872.

62. Kairuz 43. 
de Intendencia de Higiene. En 1935, el servicio se amplió para dar medicamento a quienes padecían uncinariasis. ${ }^{63}$

Una singularidad de esta campaña fue costear un capataz de peones al que se le asignaron funciones de inspector de salubridad. Este tenía a su cargo una cuadrilla de presos que se ocupaba del saneamiento de Quibdó, quienes ganaban un jornal diario de 10 centavos, el cual incluso llegó a aumentar a 15 centavos al día. El municipio también creó otro cargo de inspector de sanidad, comisionado para hacer visitas domiciliarias y garantizar de esta manera el tratamiento a una mayor porción de la población.

Si bien se hicieron grandes esfuerzos en cuestión de cobertura para llevar el tratamiento a diferentes localidades del Chocó, muchos de sus habitantes tenían cierta resistencia a los médicos, la cual era impulsada por una visión fatalista de la enfermedad. Creían que el pian era algo "propio de su raza" y que no era necesario seguir un tratamiento para eliminar las bubas. Existían también comportamientos arraigados que dificultaron las labores de los médicos. Por ejemplo, para muchos de los enfermos, iniciar el tratamiento contra las lesiones primarias o bubas "biches" era equivocado. Esto llevaba al agravamiento de las llagas y aumentaba la cronicidad de la enfermedad, a tal punto que los enfermos se presentaban al médico cuando ya su condición era muy avanzada. ${ }^{64}$

\section{Cuestionamientos éticos y la retórica de las cifras}

Con base en la experiencia del Chocó, el director de esta campaña, Antonio José Rodríguez, propuso junto al director nacional de Higiene, Arturo Robledo, una reunión para tratar el tema. Así tuvo lugar, en 1936, la Conferencia Antipiánica de Buenaventura, de la cual derivaría la nueva campaña nacional. Dentro de las conclusiones de esta reunión quedó consignado que para ese momento existían en la intendencia del Chocó y los departamentos del Valle, Cauca y Nariño, unos 70,000 enfermos de pian con la infección activa. Según declaraciones de los delegados, el 90\% de los habitantes del litoral Pacífico lo padecían. ${ }^{65}$ Producto del pliego de conclusiones de esta conferencia, se aprobó la Ley 121 de 1936, que organizaba la campaña contra el pian en el litoral Pacífico (Figura 4). ${ }^{66}$

Para su erradicación, se dio un plazo de siete años, con un gasto anual de 100,000 pesos, por un total de 759,800 pesos, que se aportarían en partes iguales entre la nación y las regiones afectadas por la enfermedad. Estos gastos contemplaban la instalación de hospitales, laboratorios y campamentos, y

63. Rodríguez R. 20.

64. Kairuz 43.

65. Kairuz 47-48.

66. "Ley 121 de 1936 por la cual se organiza la campaña contra el pian", Revista de Higiene de Bogotá (Bogotá) 1936: 126-127. 
la contratación del personal de campaña, que incluía médicos e inspectores sanitarios. ${ }^{67}$

Después de cuatro años de funcionamiento, la partida presupuestal de la campaña comenzó a agotarse y aún no se observaban avances significativos en la disminución de enfermos. Uno de los principales inconvenientes fue el retraso en el envío del medicamento hacia algunas regiones del departamento de Nariño; esto condujo a comportamientos poco éticos por parte de funcionarios de la campaña. El médico Max Llorente exponía la situación así:

Conocemos la situación en el departamento de Nariño, donde funcionan dos comisiones con nueve inspectores. Muchos meses ha habido en que estos funcionarios permanecen inactivos en sus puestos de tratamiento porque no llega el bismuto por dificultades postales, o si llega, es en cantidad exigua, de acuerdo con la partida para atender a los enfermos inscritos. Sabemos de casos en que, para evitar el justo enojo de un pianoso, después de hacer un recorrido enorme desde su casa al sitio de tratamiento, para recibir una inyección de bismuto correspondiente a su serie, el inspector que carece de ella, le aplica una de cualquiera otra sustancia para ocultar la falta. ${ }^{6}$

Gran parte del presupuesto nacional destinado a la salud se consumía en la lucha contra otras enfermedades. La lepra, dolencia terriblemente estigmatizada y que para ese momento registraba un número bajo de pacientes, consumía casi el $75 \%$ de este presupuesto, en gastos que iban desde el tratamiento, hasta la segregación en lazaretos. ${ }^{69}$ Otro caso cuestionable fue el de fiebre amarilla, enfermedad que, pese a no registrar un número importante de pacientes, capturó la atención del gobierno colombiano por solicitud de la Fundación Rockefeller, con la cual emprendió una campaña a la que destinó grandes sumas de dinero (más de 300,000 dólares).$^{70}$ Sin embargo, hacia el año 1936, el gremio médico nacional argumentaba que las campañas en Colombia debían centrarse en la lucha contra el pian antes que en la fiebre amarilla. ${ }^{71}$

Algunos funcionarios insistían en que el fracaso de la campaña contra el pian en el país había llevado a que la cantidad de pianosos ascendiera a más un millón de infectados, tal como lo afirmó en una entrevista de 1941 el médico René R. Cortés. ${ }^{72}$ Dicha cifra estaba lejos de ser cierta y buscaba impactar los espíritus. Según la conferencia antipiánica de 1936, se estimaba que había 70,000 pianosos, lo que correspondía a un 90\% de la población total de la intendencia del Chocó y de los departamentos de Cauca, Nariño yValle del Cauca. Sin embargo, el censo de 1938

67. Kairuz 49-50.

68. Max Llorente O., “Campaña antipiánica”, Heraldo Médico (Bogotá) 1941: 8.

69. Diana Obregón Torres, "The anti-leprosy campaign in Colombia: the rhetoric of hygiene and science, 1920-1940”, História, Ciências, Saúde - Manguinhos 10 (2003): 179-207.

70. Mejía Rodríguez 119-155.

71. Quevedo y otros 284.

72. "Mas de un millón de enfermos de pian en el país", Heraldo Médico (Bogotá) 1941: 29-30. 
registraba 138,817 habitantes para los núcleos poblados más importantes a lo largo del litoral Pacífico, ${ }^{73}$ por lo que, si era correcta la estimación de que el $90 \%$ de la población del litoral estaba infectada, la cantidad de bubosos de la región del país más atacada por la enfermedad era quizá cercana a 118,000. Además, al comenzar la década de 1940, el país tenía un poco más de nueve millones de habitantes, por lo que la cifra de Cortés supondría que cerca del 11\% de la población colombiana estaba afectada por esta enfermedad, lo cual demuestra un uso retórico de las cifras, pues, si bien el pian era un problema, era imposible que registrara niveles $\tan$ altos. $^{74}$

Por otra parte, médicos como Max Llorente cuestionaban la cifra de un millón y atribuían el fracaso de la campaña a la organización y a fallas o resistencia a los tratamientos convencionales. Los médicos ya dudaban de la efectividad de los medicamentos usados para combatir el pian, pues la curación no era completa, sobre todo en los casos más crónicos. ${ }^{75}$

\section{La llegada de la penicilina y la campaña antipiánica de 1950}

En la década de 1950, el pian constituía un problema de salud pública en países americanos como Brasil, Haití, República Dominicana, Guayana Británica yVenezuela, y en algunas regiones de Panamá, Ecuador, Perú y Colombia. La llegada de la penicilina durante este decenio tuvo un impacto significativo tanto en el ámbito terapéutico, como en el epidemiológico. ${ }^{76}$

A sabiendas de las dificultades que se habían observado durante diez años en la campaña, comenzaron a surgir alternativas para reorganizar la forma en que se debía combatir la enfermedad. El médico Gonzalo Guerra sugería contratar personal con mayor entrenamiento (médicos acompañados por inspectores sanitarios y enfermeras, dos por cada mil enfermos y una por cada quinientos enfermos, respectivamente) y dividir los territorios afectados en zonas o distritos de acuerdo con los focos más numerosos de pian. ${ }^{77}$ Lo más innovador de su propuesta fue cambiar la forma de tratamiento tradicional e introducir la penicilina como medida terapéutica definitiva. ${ }^{78} \mathrm{El}$ uso de la penicilina despertó un optimismo

73. Véase Contraloría General de la República, Censo General de Población, 5 de julio de 1938, ordenado por la Ley 67 de 1917, t. 6, 10, 14, 15 (Bogotá: Imprenta Nacional, 1941).

74. A propósito del abuso y retórica de las cifras para causar alarma y posicionar a la medicina diplomada en la sociedad, véase Victoria Estrada Orrego y otros, "Retórica de la cuantificación: tuberculosis, estadística y mundo laboral en Colombia, 1916-1946”, História, Ciências, Saúde Manguinhos 23.2 (2016): 277-299; Martínez y Guatibonza 179-207.

75. Llorente O. 8-9.

76. Donald R. Hopkins, "Frambesia en las Américas, 1950-1975”, Boletín de la Oficina Sanitaria Panamericana (OSP) (Washington) 1978: 262-267.

77. Gonzalo Guerra A.,"El pian en el litoral pacífico colombiano. Cómo debe orientarse su campaña”, Boletín Clínico (Medellín) marzo de 1941: 93-94.

78. Gonzalo Guerra A, “Campaña antipiánica y su tratamiento masivo-ambulatorio”, Heraldo Médico 
generalizado en los médicos, pues los resultados en el tratamiento de diferentes enfermedades bacterianas en otros países arrojaban resultados positivos.

Para el año 1947, el uso de la penicilina ya estaba extendido en Colombia y se usaba para tratar diversas enfermedades bacterianas. Al tener en cuenta su efectividad contra la blenorragia, ese mismo año el médico Laurentino Muñoz se refería a la penicilina como un medicamento "todopoderoso", y abría la puerta para su uso contra la espiroqueta causante de la sífilis. ${ }^{79}$

A la par que se proponían nuevas estrategias para el diseño de una campaña para la lucha contra el pian, en materia política Colombia iniciaba una inserción a su apertura internacional bajo el modelo de cooperación con sus aliados norteamericanos gubernamentales y privados. Esta estrategia se dio en el marco de protección continental por el impulso de los ascensos internacionales de los fascismos ligados a la Segunda Guerra Mundial. De esta forma, Colombia pasó a formar parte de una alianza internacional de países democráticos en pro de la protección continental en tiempos de guerra. Así, el país se fue adhiriendo a organismos como la Organización de las Naciones Unidas, el Fondo Monetario Internacional y el Banco Mundial, lo cual estimuló la cooperación en diferentes ámbitos, incluidos la higiene y la salud. En este sentido, la creación del Servicio Cooperativo Interamericano de Salud Pública (SCISP), desarrollado por Estados Unidos para los países latinoamericanos, jugó un papel importante en el diseño de estrategias ligadas a la contención de diferentes enfermedades que afectaban la salud pública. ${ }^{80}$

En enero de 1950, se firmó el proyecto del Convenio 116, acordado entre el ministro de Higiene de Colombia y el director del SCISP. El objeto de este convenio fue la supresión del pian como problema de higiene pública y su erradicación del territorio colombiano. ${ }^{81}$ La nueva campaña se dividió en dos planes: uno administrativo y otro científico. El plan administrativo contempló dividir las zonas de trabajo con el fin de cubrir la totalidad del litoral Pacífico y otras regiones aledañas con focos activos de pian (Figura 4). ${ }^{82}$

En cuanto al plan científico, se tuvieron en cuenta los resultados de las campañas anteriores como base para no repetir los mismos errores y se llevó a cabo la transición de los arsenicales hacia la penicilina como método de tratamiento. Se eliminó también la nomenclatura de pian primario, secundario y terciario, y se le dio prioridad a la presencia o no de lesiones para decidir aplicar el medicamento. Esta determinación se tomó para evitar errores, pues el personal que aplicaba el

(Bogotá) 1946: 25-28.

79. Laurentino Muñoz, El apogeo de las enfermedades evitables en América: paludismo, sífilis, tuberculosis (Bogotá: Ediciones de la Universidad Nacional de Colombia, 1947) 187.

80. Mario Hernández y otros, La Organización Panamericana de la Salud y el Estado colombiano: cien años de historia, 1902-2002 (Bogotá: Organización Panamericana de la Salud, 2002) 76-81.

81. Anthony J. Kranaskas y Gerardo López Narváez, "Campaña general antipiánica en Colombia”, Revista de Higiene de Bogotá (Bogotá) 1950: 265-271.

82. Alfonso Ocampo Londoño, Memoria del Ministerio de Salud Pública (Bogotá: Imprenta Nacional, 1960) 383. 
tratamiento no tenía los conocimientos científicos y técnicos necesarios para diferenciar, por ejemplo, entre pian primario y secundario. La campaña se llevó a cabo "con un mínimo de diagnóstico y aún sin él” y para inyectar el medicamento solo bastaba la noción epidemiológica. ${ }^{83}$

Una de las singularidades de esta campaña fue su movilidad, pues el personal debía movilizarse de forma activa para la búsqueda de los enfermos y no al contrario. Esto llevó a la adquisición de vehículos para el transporte fluvial. Salvo algunos núcleos urbanos, gran parte de los habitantes de la región del litoral pacífico vivían en zonas aisladas y dispersas a lo largo de los ríos y las costas. Todas las regiones que integraron el campo de acción de la campaña tenían como característica un terreno selvático, con altas temperaturas y humedad. Además, las vías de comunicación terrestre eran escasas; de allí la importancia del transporte fluvial. Los médicos de la época señalaban que la población era cercana a los 600,000 habitantes, de los cuales el 95\% era de raza negra, que había un nivel cultural muy bajo y predominaban los malos hábitos de higiene. ${ }^{84} \mathrm{Si}$ bien existían ya un tratamiento y recomendaciones en materia de higiene para la contención de la enfermedad, la imposición de estas medidas en la población se hizo dificil, pues en las zonas rurales, el pian se enmarcaba en un mundo de creencias distante y desconfiado con respecto a la medicina universitaria, las cuales reñían con la idea que tenían las autoridades sanitarias de establecer políticas y costumbres de higiene favorables a la salubridad general.

Después de diez años de funcionamiento de la campaña, el médico López Narváez concluyó que el control sistemático, las visitas repetidas a los focos de infección y la eficacia de la penicilina para la curación total de las lesiones permitieron un avance significativo en la erradicación paulatina de este problema de salud pública. Según sus propias palabras, el pian había perdido su carácter epidémico en las regiones antes azotadas por este $\mathrm{mal}^{85}$

Al igual que en Colombia, en otros países de las Américas la situación del pian cambió con el diseño de campañas sanitarias basadas en esquemas terapéuticos con la penicilina, además de las visitas domiciliarias para brindar tratamiento tanto a los enfermos como a sus contactos más cercanos. Según datos de la OPS, en los países de las Américas se dio tratamiento a 5’200,000 enfermos y contactos entre 1950 y 1960. De igual forma, el número de casos disminuyó de 19,696 a menos de 3,000 en 1960.

\section{Conclusiones}

En Colombia, las campañas antipiánicas desarrolladas entre 1925 y 1950 pusieron de manifiesto una enfermedad que atacó gran parte del país. Aunque en el imaginario social se tenía la idea de que se trataba de una patología exclusiva de las zonas costeras, su presencia fue evidente en regiones del interior del país. Si

83. Kranaskas y López Narváez 265-271.

84. López Narváez 294-342.

85. Ocampo Londoño 384. 
bien el pian presentaba una alta contagiosidad, los factores biológicos no fueron suficientes para explicar su expansión a lo largo del territorio nacional. Es claro que tanto factores sociales como culturales contribuyeron a la diseminación de la enfermedad y representaron uno de los mayores retos para los médicos, pues los comportamientos de los pianosos fueron claves para mantener activos los focos de infección. Por un lado, las pocas medidas de higiene y la costumbre de la desnudez exponían el cuerpo y abrían las puertas al microorganismo patógeno. Por el otro, la mala alimentación y enfermedades como el paludismo y la uncinariasis, endémicas en esas mismas regiones, acentuaban esta problemática.

Algunas prácticas, como trasmitir voluntariamente a los niños la infección con la premisa de que por ser de raza negra era inevitable que en algún momento de la vida contrajeran el pian, hicieron que las labores de las campañas antipiánicas fueran más arduas. Si bien existía un tratamiento específico para la curación de la enfermedad, los médicos enfrentaron imprevistos relacionados con el mal manejo terapéutico que desembocaron en la muerte de varios pianosos.

La campaña antipiánica de 1925 fue un esfuerzo inicial por tratar de contener la enfermedad en diferentes puertos fluviales. Si bien fue suspendida por una crisis presupuestal, su funcionamiento sirvió para mostrar la magnitud de una enfermedad que fue tomando grandes dimensiones en los años venideros. Para 1936, se retomaron esfuerzos por atender la problemática del pian a lo largo del litoral Pacífico. Sin embargo, la falta de dinero y la debilidad administrativa comenzaron a ser evidentes. Es probable que la falta de presupuesto llevara a algunos médicos a presentar cifras exageradas de la cantidad de pianosos en el país. Es importante resaltar también que el gremio médico nacional demandaba una mayor asignación presupuestaria a la campaña antipiánica en lugar de destinar estos recursos a la lucha contra enfermedades como la fiebre amarilla, que ya se apoyaba en dinero proveniente de la Fundación Rockefeller.

Sin duda alguna, la llegada de la penicilina como estrategia terapéutica fue crucial en la disminución paulatina de la enfermedad y se convirtió en un bastión importante en la campaña antipiánica nacional de 1950. Sumada al hallazgo de la penicilina como alternativa terapéutica de fácil uso y con alta efectividad frente al pian, la firma del acuerdo entre la nación y el SCISP fue crucial para el establecimiento de una campaña antipiánica definitiva.

\section{Fuentes}

\section{Manuscritas}

Archivo Histórico de Antioquia, Medellín (AHA)

Impresos y publicaciones

\section{Impresas}


Arango, Ernesto. “Contribución al estudio de enfermedades tropicales: pian”.Tesis de grado, Universidad de Antioquia, 1922.

Contraloría General de la República. Censo General de Población, 5 de julio de 1938, ordenado por la Ley 67 de 1917. Tomos 6, 10, 14, 15. Bogotá: Imprenta Nacional, 1941.

Delgado Uribe, Jorge. “Notas sobre el pian”.Tesis de grado en Medicina y Cirugía, Universidad Nacional de Colombia, 1928.

Kairuz, Eduardo. “Contribución al estudio del pian”. Tesis de grado, Universidad Nacional, 1938.

Mora Mora, Alberto. "Contribución al estudio de la patología y parasitología tropicales en el departamento de Antioquia". Tesis de grado, Universidad de Antioquia, 1935.

Muñoz, Laurentino. El apogeo de las enfermedades evitables en América: paludismo, sífilis, tuberculosis. Bogotá: Ediciones de la Universidad Nacional de Colombia, 1947.

Nieto Escobar, Florencio. "Algunas observaciones sobre el pian en la región de Medina”. Tesis de grado en Medicina y Cirugía, Universidad Nacional de Colombia, 1945.

Ocampo Londoño, Alfonso. Memoria del Ministerio de Salud Pública. Bogotá: Imprenta Nacional, 1960.

Vargas Cuéllar, Pedro. "El pian en la geopatología de la Costa delValle del Cauca". Tesis de grado en Medicina y Cirugía, Universidad Nacional de Colombia, 1941.

Vasco Gutiérrez, Eduardo. “Espiroquetosis cutánea (pian) y su tratamiento por el muthanol y por el storvarsol". Tesis de grado, Universidad de Antioquia, 1925.

\section{Periódicos y revistas}

Boletín Clínico (Medellín) 1941.

Boletín de la Oficina Sanitaria Panamericana (OSP) (Washington) 1927, 1932, 1935, 1978.

El Tiempo (Bogotá) 1936.

Heraldo Médico (Bogotá) 1941, 1946.

Instrucción Pública Antioqueña (Medellín) 1911, 1917.

Repertorio de Medicina y Cirugía (Bogotá) 1910.

Revista de Higiene de Bogotá (Bogotá) 1936, 1950.

Revista de la Facultad de Medicina (Bogotá) 1956.

Revista de Medicina y Cirugía (Barranquilla) 1939.

The Philippine Journal of Science (Manila) 1926.

\section{Bibliografía}


Armus, Diego y Adrián López Denis. "Disease, Medicine, and Health". The Oxford Handbook of Latin American History. Ed. José C. Moya. Nueva York / Oxford: Oxford University Press, 2010.

Biernat, Carolina y Karina Ramacciotti. Historia de la salud y la enfermedad. Bajo la lupa de las ciencias sociales. Buenos Aires: Editorial Biblos, 2014.

Estrada Orrego,Victoria y otros. "Retórica de la cuantificación: tuberculosis, estadística y mundo laboral en Colombia, 1916-1946”. História, Ciências, Saúde - Manguinhos 23.2 (2016): 277-299.

García, Claudia Mónica y Emilio Quevedo. "Uncinariasis y café: los antecedentes de la intervención de la Fundación Rockefeller en Colombia: 1900-1920”. Biomédica 18.1 (1998): 5-21.

Hernández, Mario y otros. La Organización Panamericana de la Salud y el Estado colombiano: cien años de historia, 1902-2002. Bogotá: Organización Panamericana de la Salud, 2002.

Hochman, Gilberto y otros. Orgs. Patologías de la patria: enfermedades, enfermos y nación en América Latina. Buenos Aires: Lugar Editorial, 2012.

Márquez, Jorge y Victoria Estrada. "Culebrero, tegua, farmaceuta y dentista. El Indio Rondín y la profesionalización médica en Colombia”. Anuario Colombiano de Historia Social y de la Cultura 45.1 (2018): 79-104.

Martínez, Abel y Samuel Guatibonza. "Cómo Colombia logró ser la primera potencia leprosa del mundo: 1869-1916”. Colombia Médica 36.4 (2005): 244-253.

Mejía Rodríguez, Paola. "De ratones, vacunas y hombres: el programa de fiebre amarilla de la Fundación Rockefeller en Colombia, 1932-1948”. Dynamis 24 (2004): 119-155.

Obregón Torres, Diana. Batallas contra la lepra: Estado, medicina y ciencia en Colombia. Medellín: Fondo Editorial Universidad EAFIT / Banco de la República, 2002.

. "La vacuna o la política de las cosas". Proyecto ensamblado en Colombia. Tomo 1. Ed. Olga Restrepo Forero. Bogotá: Universidad Nacional de Colombia, 2013.

. "The anti-leprosy campaign in Colombia: the rhetoric of hygiene and science, 1920-1940”. História, Ciências, Saúde - Manguinhos 10 (2003): 179-207.

Quevedo, Emilio y otros. Café y gusanos, mosquitos y petróleo: el tránsito desde la higiene hacia la medicina tropical y la salud pública en Colombia, 1873-1953. Bogotá: Universidad Nacional de Colombia, 2004.

Silva, Renán. Las epidemias de viruela de 1782 y 1802 en el Virreinato de Nueva Granada. Medellín: La Carreta Editores, 2007. 